blood mononuclear cells, this methodology may be valuable not only for the study of human genetic variants but also for providing sufficient numbers of $\mathrm{mDC}$ for the study of human myeloid dendritic cell function more generally.

Acknowledgements This work was supported by grant AI30199 from NIH/NIAID (to I.R.R.).

\section{II-04 BONE MARROW MESENCHYMAL STEM CELLS FROM PATIENTS WITH SLE MAINTAIN AN INTERFERON SIGNATURE DURING IN VITRO CULTURE}

${ }^{1}$ Lin Gao, ${ }^{1}$ Mary OConnell, ${ }^{1}$ Maria Allen, ${ }^{2}$ Andrew McDavid, ${ }^{1}$ Jennifer H Anolik, ${ }^{1}$ Richard J Looney*. 'Department of Medicine, University of Rochester School of Medicine and Dentistry, Rochester New York, USA; ${ }^{2}$ Department of Biostatistics and Computational Biology, University of Rochester School of Medicine and Dentistry, Rochester New York, USA

\subsection{6/lupus-2018-Ism.103}

Background We have previously shown that SLE BMSCs have a pro-inflammatory mediated by a MAVS and IFN $\beta$ feedback loop. Compared to healthy controls, SLE BMSCs produced increased amounts of IFN $\beta$ and had increased mRNA for several genes induced relatively specifically by IFN $\beta$. They also had decreased proliferation, increased ROS, increased DNA damage and repair (DDR), a senescence associated secretory phenotype, and increased senescence-associated $\beta$-galactosidase. To better understand the phenotype of SLE BMSCs we conducted RNA sequencing.

Methods Patients fulfilling SLE classification criteria and age and sex matched healthy controls were recruited under an Institutional Review Board approved protocol (6 pairs). Bone marrow aspirates and peripheral blood samples were obtained. BMSCs were isolated with low density Ficoll/Hypaque and grown in tissue culture. Purity of BMSC cultures were verified by flow cytometry. Early passage BMSC were harvested and mRNA samples were sent for RNAseq through the University of Rochester Genomics Core. Serum samples with assayed for IFN $\beta$ using an ELISA from PBL.

Results Hierarchical clustering of normalized RNAseq profiles found SLE patients with high levels of serum IFN $\beta$ ( $>13$ units per XXX) grouped together while SLE patients with low levels of IFN $\beta$ grouped together with healthy controls. Principal component analysis found the majority of the variance could be explained by PC1 (32.3\%) and PC2 (25.5\%). While PC1 did not separate SLE or SLE IFN $\beta$ high from the other samples, PC2 clearly differentiated SLE IFN $\beta$ high samples from SLE IFN $\beta$ low and control samples. SLE IFN $\beta$ low and control sample overlapped in both PC1 and PC2. The top upregulated genes in PC2 were RSAD2, MX1, IFIT1, IFIT2, OAS1, CMPK2, OASL, IFIT3, ISG15, IDO1, IFI6, MX2, HERC5, and IFIH1 ... all type I interferon-induced genes.

Conclusions BMSCs from SLE patients are heterogeneous. A subgroup of SLE BMSC are distinguished from other SLE BMSC and from controls by increased levels of mRNAs induced by type I interferons. Moreover, SLE BMSC with increased levels of mRNA for type I interferon-induced genes when grown in vitro are derived from patients with increased levels of IFN $\beta$ in vivo.

Acknowledgements This work was supported in part by a grant from the Lupus Research Alliance.

\section{II-05 B CELLS FROM SLE PATIENTS HAVE INCREASED ENDOGENOUS PRODUCTION OF IFN $\beta$ WHICH IS STIMULATED BY BCR SIGNALING AND IS REQUIRED FOR SURVIVAL OF AUTOREACTIVE B CELLS}

'Shanrun Liu, 'PingAr Yang, 'Qi Wu, ${ }^{1}$ Bao Luo, ${ }^{1}$ Ojo, Oluwagbemiga A, ${ }^{1}$ William A Essman, 'Jennie A Hamilton, 'Jun Li, 'W Winn Chatham, ${ }^{1}$ Hui-Chen Hsu, ${ }^{1,2}$ John D Mountz* . 'Department of Medicine, University of Alabama at Birmingham, Birmingham, $A L ;{ }^{2}$ Department of Medicine, Birmingham VA Medical Center, Birmingham, AL

\subsection{6/lupus-2018-Ism. 104}

Background Increased type I interferon (IFN) has been shown to affect survival and activation of B cells in SLE. This study investigated novel mechanisms of endogenous production and autocrine activity of IFN $\beta$ in SLE B cells at the single-cell level.

Methods IFN $\beta$ in B cells from SLE patients was analyzed using t-SNE platform based high dimensional flow cytometry. Intracellular IFN $\beta$ expression was visualized and analyzed by super-resolution confocal imaging and ImageStream analysis. Single cell gene expression analysis was carried out using the Fluidigm/BioMark system for targeted expression of low abundance genes, and the $10 \mathrm{x}$ Chromium platform for unbiased transcriptome analysis of up to $4,000 \mathrm{~B}$ cells per subject. Functional production of type I IFNs by B cells was analyzed using a human type I IFNs SEAP reporter HEK293 cell line. Results High dimension flow cytometry analysis identified intracellular IFN $\beta$ expression in pDCs, B cells, and CD4 T cells. B-cell endogenous IFN $\beta$ was required for optimal in vitro BCR and TLR7-induced activation and survival of $\mathrm{B}$ cells. Using a Fluidigm targeted-gene approach, B cells could be divided into type I IFN expressing (IFN+) or type I IFN stimulated gene $(I S G+)$ subpopulations, suggesting B cells not only respond to type I IFNs but also express type I IFNs including IFNB and different IFNA genes. TLR7 and TLR3 were mainly expressed by IFN + cells whereas TLR9 was mainly expressed by $I S G+\mathrm{B}$ cells. The production of functional IFN $\beta$ and IFN $\alpha$ protein by single B cells from SLE subjects and was verified using a novel alkaline phosphatase live staining of HEK-blue reporter cells. There was enhanced IFNAR signaling by reporter cells in direct contact with SLE B cells which was blocked by anti-IFN $\beta$ and anti-IFN $\alpha$. Interesting, anti-Ig crosslinking was required for optimal B-cell endogenous type I IFNs to stimulate responder cells. Unbiased single cells transcriptome analysis of SLE B cells using the 5' 10X Chromium platform and Loupe V(D)J Browser indicated that gene clusters in type I IFN expressing or responding SLE $\mathrm{B}$ cells exhibited unique heavy- and light-chain gene expression repertoires.

Conclusion (i) B cells are an important source of type I IFNs in modulating TLR and BCR responses in SLE; (ii) well-orchestrated and distinct programs in type I expression and responses genes in subsets of B cells, and (iii) distinct pathways of B cell survival and activation based on combined signaling through BCR, TLR, and IFNAR with a distinct BCR heavy- and light-chain repertoire.

Acknowledgements This work was supported by grants from R01-AI-071110, R01 AI134023, Lupus Research Alliance Distinguished Innovator Award, I01B $\times 004049$, and $1 \mathrm{I} 01 \mathrm{~B} \times$ 000600 to J.D.M, 2T32AI007051-39 Immunology T32 Training Grant and the LFA Finzi Summer Fellowship to J.A.H, and the LRA Novel Research Award to H.-C.H. 